\title{
L'Insuffisante différenciation fonctionnelle du Droit au Brésil \\ et le exemple canadien: le Droit fondamental à la santé dans la \\ Constitution Fédérale de 1988.
}

\section{The Insufficient Functional Differentiation of Law in Brazil and the Canadian Example: The fundamental right to health in the Federal Constitution of 1988}

Germano Schwartz ${ }^{1}$

Submetido em 23 de julho e aprovado em 30 de julho de 2017.

\begin{abstract}
Résumé: Cet article vise, sur la base de l'exemple canadien, démontrent, de la théorie des systèmes sociaux autopoḯtiques appliqués au Droit (Luhmann), la différenciation fonctionnelle insuffisante du droit à la santé au Brésil. Comme laps de temps, adopte la promulgation de la Constitution de 1988 au Brésil pour défendre la thèse selon laquelle le problème central de l'efficacité du droit à la santé sur les résultats du sol brésilien du fait que la loi souffre influence extérieure sans préserver leur autonomie, fait diversifiée ce qui se passe au Canada.
\end{abstract}

Mots-Clés: Droit a la Santé, Brésil, Canada, différenciation fonctionnelle

\begin{abstract}
Based on the Canadian example, this article aims to demonstrate, from the theory of autopoietic social systems applied to Law (Luhmann), the insufficient functional differentiation of the right to health in Brazil. As a temporal cut, it adopts the promulgation of the Federal Constitution of 1988 in Brazil to defend the thesis that the central problem of the effectiveness of the right to health on Brazilian soil results from the fact that the Law suffers external influence without preserving its autonomy, which happens in Canada.
\end{abstract}

Keywords: Right to Health, Brazil, Canada, Functional Differentiation.

\section{Forme d'approche}

Promulguée le 12 octobre $1988,{ }^{2}$ la constitution de la République fédérative du Brésil a représenté, symboliquement, un nouveau moment pour les Brésiliens. En effet, toute une série de sentiments et de désirs réprimés pendant les années de dictature militaire y ont été inscrits. ${ }^{3}$ 
Il était donc naturel que les attentes normatives projetées par la société brésilienne se dirigent vers une (ré)orientation du pays. En ce sens, par exemple, le texte constitutionnel contient une liste abondante de droits fondamentaux, ce qui est tout à fait logique si l'on comprend cette positivation comme une réaction aux actes de torture ${ }^{4}$ et de restriction des droits fondamentaux pratiqués sous l'égide de la Constitution fédérale de 1967.

Le souci de rompre avec le passé était si fort que cette Charte contient 345 articles (250 dans le texte et 95 dans les Actes des dispositions constitutionnelles transitoires) dont beaucoup ne peuvent pas être classés, sur les bases de la théorie de la Constitution, comme ayant un caractère matériellement constitutionnel. Ils y figurent toutefois comme une espèce de garde-fou hierárquico-normatif, une tactique législative discutable, mais compréhensible dans le contexte social brésilien.

Surnommée, donc, «Constitution citoyenne», elle représentait en soi l'espoir d'un peuple aspirant au développement et à la justice sociale. ${ }^{5}$ Elle a également servi de transition paradigmatique vers une nouvelle posture par rapport à la mise à effet des droits fondamentaux via le pouvoir judiciaire. Autrement dit: elle a refait du Brésil un État démocratique de Droit, ${ }^{6}$ et a donc pris sur soi une fonction active de la société brésilienne.

Cependant, 20 (vingt) ans après, le fait est que le texte original a été profondément modifié par 56 Amendements constitutionnels et une révision. Plusieurs de ces changements ont eu pour toile de fond la discussion entre les coûts des droits prévus et le besoin de déréglementation de certains secteurs. ${ }^{7}$ En tant que pays périphérique, le Brésil a été acculé par les forces de la mondialisation.

Le phénomène mondialisateur a produit plusieurs formes de pression sur la Constitution brésilienne, dont celle du système économique sur le système juridique, qui a fortement pesé sur les modifications de la Loi fondamentale, et qui est justement l'objet d'étude de cet article.

Il en est de même avec le Canada? Notez que le droit des deux pays ont de nombreuses différences (DOS SANTOS, 2007), par exemple, l'existence de vingt-cinq constitutions au Canada. Le droit à la santé canadien, cependant, est régie par une loi applicable au niveau national, tout en préservant les particularités régionales reconnues dans le système des fonds publics de transfert pour chaque province. Il est la Loi sur la santé du Canada (1985). 
Alors que le Brésil occupe le numéro 125 dans le classement mondial de la santé publique, le Canada est en trentième lieu (Tandon, et.al, 2017). Ces données démontrent les différences régionales entre les deux pays ont signalé par Cattoni (2006). De plus, il révèle la nécessité de rechercher les similitudes et les différences possibles entre les deux systèmes - la santé et les droits (LEAL, CABRAL et Perreault, 2010)-.

Pour anticiper le point central de cet essai: il faut que le système juridique des pays périphériques conserve son autonomie par rapport à l'ouverture cognitive exercée par la communication provenant principalement du système économique. En fait, l'empiètement du code de ce dernier (Avoir/Ne pas avoir) sur celui du premier (Rech/Unrecht - Droit/ non droit) a causé, au Brésil, une réalité déjuridifiante de la Constitution brésilienne (et des droits sociaux) dans ses 20 années d'existence.

\section{La Constitution de 1988: formation du sens constitutionnel au Brésil.}

L'histoire constitutionnelle brésilienne n'a pas été favorable à la formation d'une volonté de respect pour la Constitution, car il y a eu sept chartes avant la promulgation du texte constitutionnel actuel. Une courte digression nous permettra de vérifier que ces changements ont présentaient une certaine continuité, brisée par l'adoption d'une Loi fondamentale à caractère antidémocratique et suivie par celle de 1988, qui l'a prise à contre-pied, en un véritable mouvement de pendule.

Quand, fuyant 1'invasion des armées napoléoniennes, en 1808, la famille royale portugaise est venue s'installer au Brésil, ce pays a acquis un statut différent, puisqu'il cessait d'être une colonie pour devenir un Royaume uni. Néanmoins, les lois étaient portugaises (comme le monarque), et aucune Loi fondamentale ne tenait compte des caractéristiques de la société brésilienne de l'époque.

Le 07 septembre 1822, le Brésil a proclamé son indépendance de l'ancienne métropole pratiquement sans heurt, ce qui, d'une certaine manière, a beaucoup influencé la formation du sens du peuple brésilien, beaucoup plus attaché à la négociation qu'au

conflit. Dans l'esprit de l'époque et à l'instar des autres pays d'Amérique, comme il s'agissait d'une nouvelle nation, le besoin d'un changement de paradigme s'est fait sentir. Est-ce que la Constitution a reflété ce nouvel esprit? 
Non. Le Brésil «a choisi» de continuer à être un empire et la raison en était simple: l'indépendance avait été proclamée par le fils de l'Empereur portugais qui est devenu le nouveau monarque. En 1824, la première constitution brésilienne promulguée était donc celle d'un empire et elle maintenait les mêmes bases de la société bourgeoise brésilienne qui avait surgi avec le Brésil colonie et s'était renforcée pendant le Royaume uni. Il fallait maintenir le status quo.

De manière inédite, le Brésil consacrait un quatrième pouvoir, Modérateur, qui travaillait côte à côte avec les autres pouvoirs institués (Exécutif, Législatif et Judiciaire). Le monarque se réservait toutefois la capacité de réviser les actes des autres pouvoirs, et même de substituer leurs membres.

Le changement a été lent. L'abolition de l'esclavage, en 1888, a été le point culminant de la transition d'un empire vers une république. De nouveau, cela s'est passé pratiquement sans heurts. Le 15 novembre 1889, à l'instar des États-Unis d'Amérique et sous l'influence directe de ce pays, le Brésil est devenu la République des États-Unis du Brésil.

En 1891, fruit d'une Commission de notables, une nouvelle constitution du Brésil a été promulguée. Bien qu'elle ait pris de nets contours libéraux, elle ne rompait pas avec le passé. Le pouvoir modérateur a évidemment été aboli, mais le vote n'était pas accordé à tous les Brésiliens, ${ }^{8}$ puisque seule une partie d'entre eux pourrait faire usage de cette prérogative, en une déviation typiquement brésilienne des institutions démocratiques.

À l'époque, le Brésil était une nation agricole dont les produits les plus importants étaient le café (São Paulo) et le lait (Minas Gerais). Ces deux états, qui étaient les plus densément peuplé, étaient donc aussi les plus importants du point de vue économique. Comme les Présidents de la république provenaient, en alternance, de São Paulo et de Minas Gerais, cette politique est restée fameuse sous le nom de «café com leite» (café au lait).

Le krach de 1930 et le dissidences internes des états ignorés par la politique en vigueur ont fait s'écrouler la valeur économique du café et du lait, et donc, la force politique des états de São Paulo et de Minas Gerais. Une nouvelle Constitution a été promulguée en 1934, dont la grande figure était Getúlio Vargas, né dans l'état du Rio Grande do Sul, personnage politique qui centraliserait les mouvements constitutionnels suivants. 
Quand la IIe guerre mondiale a éclaté, Getúlio Vargas, sympathisant de l'axe, était président du Brésil. En 1937, sous prétexte du besoin de mettre en œuvre des réformes sociales (ce qu'il a vraiment fait), barrées, selon lui, par les forces économiques du passé, il a décrété la dissolution du Congrès et a substitué plusieurs magistrats. En outre, il a octroyé la Constitution de 1937, dont l'inspiration totalitaire était telle qu'elle est passée à la postérité sous le nom de Charte polonaise, en une allusion claire à la Loi fondamentale en vigueur en Pologne. ${ }^{9}$

À la fin de la guerre, en 1945, bien que le Brésil ait lutté aux côtés des alliés (1943) et ait participé à des combats dans le sud de l'Italie, il a commencé à être vu comme un dirigeant dépassé. En 1946, le Brésil a adopté une nouvelle Constitution dont le caractère libéral a permis une courte période d'élections présidentielles démocratiques. C'est à cette époque qu'ont surgi des figures politiques comme Jânio Quadros et Juscelino Kubitschek.

En raison des vents démocratiques et de la pluralité de partis consacrée par la constitution de 1946, des idéaux marxistes se sont répandus au Brésil, comme dans tous les pays d'Amérique du sud et des partis «communistes» comme le P.C. do B.10 et le P.C.B. ${ }^{11}$ ont eu leur importance dans la vie politique. Il y existait donc un «danger» socialiste.

C'est ce que les militaires avaient à l'esprit, en 1964, lorsqu'ils ont pris le pouvoir, dissolu le Congrès et occupé le poste de Président de la république. En 1967, ils ont proclamé une constitution antidémocratique, qui instituait le vote indirect pour la présidence. Elle a été endurcie plus encore par plusieurs «Actes institutionnels»* et un Amendement constitutionnel (1969). La censure préalable, par exemple, a commencé à être de règle pour tous les moyens de communication.

Notons également que, à cette époque, les mouvements sociaux s'opposant au régime en vigueur ont été rares et sporadiques. Un changement ne surviendrait qu'en 1984, quand la société brésilienne en masse a commencé à souhaiter voter pour élire le Président de la république, en une réaction claire contre la politique en vigueur.

Cette brève récapitulation historique n'a nulle intention de détailler l'historicité des événements mais de démontrer que tous les changements constitutionnels, exception faite de celui qui a conduit à promulgation de la Constitution de 1988, étaient insérés dans un contexte économique. Au Brésil, la formation du sens d'une Loi fondamentale est si 
inexorablement liée à l'économie que, à plusieurs reprises, celle-ci a pris le dessus sur le sens juridique de la Constitution.

Cela explique pourquoi les Cours et tribunaux, les opérateurs et les citoyens n'aient jamais vraiment vu la Loi fondamentale d'un bon œil pendant la période du Brésil en tant que nation. Le changement de paradigme a été introduit par la Constitution de 1988, fruit d'un mouvement politique d'opposition au régime en vigueur qui n'en a pas moins laissé quelques restes: le code économique (Avoir/Ne pas avoir) a resurgi en force ces 20 dernières années, qui défigure la formation du sens de cette loi et, comme nous l'avons dit, cause sa réalité déjuridifiante.

\section{La Constitution de 1988: formation du sens du texte constitutionnel.}

En 1982, le Brésil a réalisé des élections, exception faite pour le poste de Président de la république. En 1985, le Collège électoral a élu un civil à la présidence, Tancredo Neves, qui est mort avant de prendre ses fonctions. Après une longue discussion juridique, le vice-président, José Sarney, également civil, a fini par assumer la présidence et des élections pour le Congrès national se sont tenues. Le nouveau président a ensuite convoqué une commission connue sous le nom d' «Afonso Arinos» ou «Commission des notables», composée par 50 grands juristes.

En un message au Congrès national, transformé en l'amendement constitutionnel 26/85, Sarney a convoqué le Congrès national à jouer le rôle d'Assemblée constituante. Il convient de noter que, comme la constitution de 1967, qui ne dit rien sur la possibilité de convoquer une Assemblée constituante, était encore en vigueur, cet amendement était truffé d'inconstitutionnalités. Cependant, il se légitimait par la volonté du peuple, exprimée lors de la campagne nationale pour les élections directes (Diretas já - 1984), exigeant que tous les citoyens brésiliens ait le droit de voter directement pour la Présidence de la république.

Dans de ce contexte, la Constitution de la République fédérative du Brésil, également connue comme «Constitution citoyenne», nom que lui avait donné Ulysse Guimarães, alors membre du congrès, spécialement en raison de la liste de droits fondamentaux (sociaux) qu'elle prévoyait, a été promulguée le 05 octobre 1988. 
Or, cette Constitution présente un dualisme: elle est tantôt sociale, tantôt libérale. Son texte reflète la force des deux grands groupes politiques ayant participé aux discussions lors de la Constituante: le Centrão (grand centre), de droite, aligné sur le status quo ante et l'Esquerda (gauche), qui abritait un large éventail d'adversaires du régime militaire. Comme toujours dans l'histoire brésilienne, la constitution de 1988 a avant surgi d'un vaste accord.

Cela n'a cependant pas empêché qu'elle contienne une série d'avancées. Elle a adopté un texte analytique essentiellement par crainte de ce qu'une Charte légale générique puisse évider son sens. A l'époque, il fallait que la Loi fondamentale soit un instrument qui dirige la société brésilienne.

En gros, la Constitution de 1988 peut être divisée en trois:

(a) Une Constitution sociale, qui met en application les droits de plusieurs générations. Certains ont été récupérés (libre expression), d'autres renforcés (droit au travail), et plusieurs autres (droit à la santé, droit environnemental) ont été positivés de manière inédite.

(b) Une Constitution politique, qui établit la tripartition des pouvoirs comme règle fondée sur l'harmonie et l'indépendance entre exécutif, législatif et judiciaire. En ce sens, elle a conservé comme clause fondamentale le droit au vote secret et régulier et elle a également structuré la forme d'État (fédération) et de ses entités, délimitant leur autonomie respective.

(c) Une Constitution économique, qui respecte la propriété privée et la libre initiative, basées, toutefois, sur une notion de justice sociale et de respect du travail qui traverse son sens.

Un reflet de cette orientation triangulaire en est qu'elle a destiné un chapitre entier à l'ordre social. En outre, plusieurs droits sociaux ont été établis dans son article 6e, comme la sécurité, le travail, la santé, la prévoyance, etc..

En même temps, sur le plan politique, tout attentat à l'ordre démocratique a été qualifié comme crime sans droit à une mise en liberté sous caution, bloquant ainsi tout possibilité de coup d'État, outre le droit, déjà cité, au vote direct pour toutes les niveaux des pouvoirs exécutif et législatif. 
Et finalement, sur le plan économique, la propriété privée s'est vue imposer une nouvelle limite: la fonction sociale. Le Statut de la Ville dérive directement de la détermination constitutionnelle cherchant à combiner les intérêts économiques immobiliers et le développement environnemental. La libre initiative a toutefois été préservée de manière assez ample.

Par ailleurs, du point de vue structurel, à l'exception du préambule, elle a été divisée en 10 chapitres, dont la position géographique a intentionnellement été bien différente de la constitution militaire jusqu'alors en vigueur, puisqu'elle est organisée ainsi: (1) Principes fondamentaux, (2) Droits et garanties individuels, (3) Organisation de l'État, (4) Organisation des pouvoirs, (5) Défense de l'état et des institutions démocratiques, (6) Taxation et budget, (7) Ordre économique et financier, (8) Ordre social, (9) Dispositions constitutionnelles générales et (10) Acte des dispositions constitutionnelles transitoires.

Néanmoins, sa caractéristique dominante, comme nous l'avons déjà souligné, est le désir de démocratie, et donc de changement vers un État démocratique de droit basé notamment, selon son article troisième, sur les valeurs d'une société libre, juste et solidaire, dont les buts majeurs sont l'éradication de la pauvreté et de la marginalisation, et une réduction des inégalités sociales et régionales. Mais cela persiste-t-il? Une question reste en suspens: les vents de 1988 soufflent-ils encore en 2017 ?

\section{Les coûts des droits sociaux et la réserve du possible: la déviation du sens de la Constitution de 1988.}

La formation du sens de la Constitution fédérale de 1988, telle qu'elle a été définie ci-dessus, conduit à deux conclusions: (a) elle a opté pour un changement concernant les droits fondamentaux, auxquels son texte réserve une place de choix; (b) elle a cherché une transformation paradigmatique de la société brésilienne par son option en faveur d'un État démocratique de droit.

Cependant, comme ces deux positions exigent des ressources pour se concrétiser, elles sont donc devenues des tâches contractuelles de l'État brésilien, notoirement en manque de ressources financières face à l'importante mission de protéger les droits 
fondamentaux. Il est donc naturel, lors de sa mise en œuvre et de son assimilation par les acteurs sociaux, que cette dualité (coûts x droits) ait assumé une grande importance.

C'est particulièrement en ce qui concerne les droits sociaux prévus dans la Constitution de 1988 que la thématique de la Réserve du possible a été mise en relief dans le cadre de discussions universitaires et jurisprudentielles au Brésil. il est indéniable que la toile de fond de ces débats met en jeu l'influence de l'économie - et de son autopoïèse - sur le Droit.

La théorie de la Réserve du possible part du principe que, pour satisfaire certains droits sociaux - à caractère de prestation (Art. $6^{\circ}, \mathrm{CF} / 88$ ) -, il faut disposer de ressources, lesquelles sont toutefois limitées. Ainsi, selon Krell, ${ }^{12}$ l'élévation du niveau de leur réalisation serait toujours conditionnée par le volume de ressources susceptibles d'être mobilisées à cet effet. Il y a donc une liaison claire entre le système juridique (droit à la santé) et le système économique (ressources) et il importe de savoir comment se passe l'autopoïèse de ce sous-système, car elle peut dévoiler l'appareil interne de cette question et son prétendu couplage structurel sur le droit.

Non par hasard, le point de départ, ou problème initial de l'économie, est la pénurie. ${ }^{13}$ Autrement dit: certains biens ne sont disponibles qu'en quantité limitée et l'accès des uns à ces derniers exclut celle des autres. Ainsi, le paradoxe du système économique se fonde sur le fait que la tentative d'éliminer la pénurie va engendrer d'autres formes/ modalités de pénurie, l'inclusion générant donc l'exclusion. ${ }^{14}$

Selon Luhmann, ${ }^{15}$ l'économie peut être décrite comme un système de paiements qui sont temporaires et très vite remplacés, sans appel, par d'autres paiements. Il n'y a donc paiement que parce qu'il existera d'autres paiements, ceux-ci dépendant de ceux-là et vice-versa.

Cette condition de reproduction du système économique n'est faisable que par l'intermédiaire d'une création sociale, l'argent, qui, en fait, se transforme en désir mimétique ${ }^{16}$ également réglementé par le Droit. L'argent devient un moyen de communication symboliquement généralisé. Le crédit joue également cette fonction (rendre des paiements faisables), car il acquiert une mémoire sélective par rapport à l'argent (qui paie/qui ne paie pas). 
La circulation d'argent permet les paiements et, donc, une généralisation matérielle, temporelle et sociale ${ }^{17}$ de possibilités applicables au système économique, qui ne se fixe à aucun moment donné dans le temps, garantissant ainsi son autoreproduction. Voilà pourquoi le marché financier devient omniprésent, et, pour Luhmann, il peut se comparer à un Dieu ${ }^{18}$ qui a une influence sur les décisions et les oriente, même s'il n'est pas matériellement présent.

Dans cette ligne de raisonnement, l'autopoïèse du système économique, comme pour tous les autres sous-systèmes sociaux, passe par une fermeture opérationnelle (paiement) et une ouverture cognitive (besoin). Une donnée issue d'un autre système - les droits sociaux -, qui doivent être filtrés face à la logique du système économique, s'insère dans cette constellation communicationnelle. Il s'agit de besoins, puisque cette logique est comprise comme tout ce qui peut s'orienter à partir d'une certaine production. ${ }^{19}$ (ex: industrialisation des médicaments et les lois de propriété intellectuelle).

Ce que nous venons d'exposer montre que la fonction du système économique n'est pas de satisfaire des besoins (droits sociaux), mais bien plutôt de garantir que la satisfaction des besoins ${ }^{20}$ actuels n'interviendra pas dans la création et/ou la fourniture de besoins futurs. Donc, la satisfaction totale est une impossibilité qui bloque la continuité du système économique.

En outre, le système économique possède un centre et une périphérie et, comme il est organisé dans une société financière mondiale, les décisions sont prises dans certains endroits avant de s'irradier vers d'autres. Le centre vit de la demande de la périphérie (besoin), ce qui lui permet d'étendre le crédit et, par conséquent, les possibilités de liquidité. C'est un cycle continu autorécursif. Or, le Brésil, qui est situé dans la périphérie, ${ }^{21}$ dépend du crédit que les pays centraux veulent stimuler. Comment penser les droits sociaux fondamentaux des Brésiliens à partir de ces présupposés? C'est dans la périphérie que le système économique trouve des couplages structurels pour les besoins orientant l'économie. ${ }^{22}$

Effectivement, l'allocation de ressources est une forme de paiement. Même quand la décision de dépense provient du système politique, ${ }^{23}$ l'argent pour la protection juridique des droits sociaux, dans la théorie de la réserve du possible, est indéniablement 
une décision issue du système économique, passant par ses structures. Ce modèle est défendu par un mouvement de Droit dénommé Law and Economics, dont le principal représentant, Richard Posner ${ }^{24}$, est bien clair quand il affirme, par exemple, que même pour la santé, les ressources (donc, les paiements) sont limitées. Au Brésil, Gustavo Amaral, ${ }^{25}$ qui suit cette même ligne, affirme que les relations dans le champ de la santé sont également économiques, car

le professionnel de santé commercialise des marchandises et des services, le patient est le consommateur et l'acheteur peut être le patient, son assureur, son employeur ou le gouvernement. Les soins médicaux sont des produits offerts sur un marché répondant aux lois de l'économie.

Voilà donc l'autopoïèse du système économique et la logique de son action dans la question de la réserve du possible. Ainsi, des choix doivent être faits, qui ne peuvent être qualifiés de tragiques que si l'autopoïèse du système économique dicte l'autopoïèse du système juridique, en une interférence qui ne préservera la fermeture opérationnelle d'aucun de ces deux sous-systèmes sociaux différents. La question est donc de savoir comment le Droit, dans son autopoïèse, perçoit de telles communications et les juridicise.

Si les droits sociaux forment un besoin (ouverture cognitive) pour le système économique, pour le système juridique ils constituent un élément décisoire de sa structuration interne en tant que droit fondamental de la Constitution brésilienne. Il est donc naturel qu'il existe une relation de coévolution entre ces deux systèmes. Il s'agit donc de savoir comment le système juridique garantit son autoreproduction à partir de la pression exercée par le système économique.

Selon Hutter $^{26}$, du point de vue du système juridique, la valeur économique est une source de recherche de justice alors que, pour le système économique, la conversation avec le Droit pousse à des changements dans les messages économiques. Donc, dans l'Économie, la justice est perçue comme une recherche de valeur.

En ce sens, bien que, chez Luhmann, l'autopoïèse soit un projet radical, il n'y a aucun doute que la proposition de Clam d'une autopoïèse spécifique du Droit est correcte, car elle dérive de l'autopoï̀se du système social qui ne parvient pas, pour d'innombrables raisons, à éviter cette évolution. Selon ce dernier auteur, le système juridique assume la 
tâche de transformer formellement les faits mondains dont l'appréhension cognitive ne peut fournir qu'une observation exempte de valeur, qui reproduit, dans son intention, des interventions normatives. ${ }^{27}$

Voilà le présupposé d'analyse de la réserve du possible dans le droit: l'autopoïèse du système juridique doit observer les faits du monde comme transformables. Ce ne seront jamais des données prêtes. Les interventions normatives (actes, décisions, lois) sont administrées par une ouverture cognitive qui doit être retraitée dans la logique du système juridique (transformation des faits mondains - pénurie de ressources).

La théorie de la réserve du possible a été mentionnée par le Tribunal constitutionnel fédéral allemand lors du jugement du cas Numerus Clausus I, expression qui, selon Leivas $^{28}$, date de 1960 et prétendait limiter numériquement l'accès des étudiants à certains cours supérieurs (droit, médecine, pharmacie, entre autres). Ceux qui n'ont pas été reçus ont postulé judiciairement le libre accès à l'exercice et au choix de la profession, mais ils ont été déboutés en raison de la nature déjà citée de la théorie de la réserve du possible (pénurie de ressources). Même s'il s'agit sans aucun doute d'un besoin fort différent de celui existant par rapport aux droits sociaux au Brésil, cette information doit être prise en considération quand le système juridique est provoqué à décider à ce sujet.

Le Droit autopoiétique se meut sur une unité distinctive (Recht/Unrecht). ${ }^{29} \mathrm{C}$ 'est un système fermé opérationnellement en même temps qu'il est ouvert cognitivement. Il ne fonctionne pas sur des fondements de validité ou de symétrie. Pour le système juridique, il importe de définir ce qui est Droit et ce qui ne l'est pas.

Les opérations internes du système juridique (jurisprudence, communications, discours juridiques, etc..) ont besoin de structures (Loi, Pouvoir judiciaire) pour que soit prise une décision disant ce qui est Droit et ce qui ne peut pas être considéré comme tel. Surgit alors le besoin d'un code propre et fonctionnellement différencié. ${ }^{30}$ C'est le Recht/ Unrecht, forme d'acquisition évolutive spécifique du Droit. Aucun autre sous-système partiel de la société n'opère avec cette unité décisoire distinctive ou ne pourrait le faire.

Le système juridique est donc dynamique, puisqu'il est orienté vers une décision fonctionnellement équivalente. ${ }^{31}$ Le code est l'autre côté de sa programmation. Il promeut des sélections forcées, réduisant ainsi la complexité issue de la double contingence 
(attentes d'attentes). A partir d'alors, les attentes normatives se maintiennent sur des niveaux socialement acceptables.

La condition décisoire du système du droit est un paradoxe: le Recht est décidé sur les bases de l'Unrecht. Le Droit d'une partie est le non Droit d'une autre. Cet antagonisme est exclus par des conditionnements (lois). D’autres codes sont gérés, qui sont établis par l'organisation de chaque système (constitutionnel/inconstitutionnel). Les Codes sont des manières de se détacher du paradoxe. Ce sont les conditions de possibilité de conditionnements qui règlementent quelle valeur sera valablement appliquée (droits sociaux ou réserve du possible).

Néanmoins, la réserve du possible est un point de contact périphérique qui produit des bruits de fond devant être nécessairement filtrés par le système juridique, même si cela conduit à la reproduction d'une tautologie: le non Droit (réserve du possible) évalué par le Droit (santé) révèle que la négation renforce la positivité (droits sociaux). Toutefois, les tribunaux agissent selon leurs codes communicationnels et organisationnels (obligation de décider, position centrale et hiérarchisée) pour redistribuer au reste du système sa récursivité décisoire.

Dans cette ligne de raisonnement, selon les Cours et les Tribunaux brésiliens, il est impossible d'appliquer le raisonnement du système économique (pénurie de ressources) à un droit social comme la santé, compris du point de vue du système juridique (droit fondamental), puisque cela reviendrait à renier la nature même du droit (décider sur les bases de son autopoïèse spécifique). Sinon, le Droit serait tout sauf le Droit. Telle est la position monocratique d'Aires , par exemple: ${ }^{32}$

En outre, la santé est un droit social fondamental, à propos duquel l'on ne saurait argumenter avec le principe de la «réserve du possible» car soit l'on accomplit un minimum, un noyau existentiel du droit, soit l'on installe, en dernière analyse, l'arbitrage de l'État, ce qui est intolérable.

De même, dans le compte rendu d'un jugement récent de la Cour supérieure de Justice, ${ }^{33}$ la ministre Eliana Calmon affirme qu'il est impossible que la théorie de la réserve du possible soit appliquée aux droits à la santé et à la vie, car elle considère que tous deux sont des biens maximums et qu'une telle préoccupation ne saurait être remise à plus tard. 
La Cour suprême fédérale admet également que la réserve du possible ne peut pas être une communication externe et qu'elle doit être filtrée par le système juridique comme Droit. C'est la position du ministre Celso de Mello: ${ }^{34}$

Il ne semble toutefois pas licite au pouvoir public, dans cette hypothèse - au moyen d'une manipulation indue de son activité financière et/ou politico-administrative - de créer un obstacle artificiel révélant le propos illégitime, arbitraire et censurable de frauder, de frustrer et d'empêcher l'établissement et la préservation, en faveur de la personne et des citoyens, de conditions matérielles d'existence minimums. Il convient donc d'aviser que la clause de la «réserve du possible» - sauf en l'occurrence d'une raison juste et objectivement vérifiable - ne peut pas être invoquée par l'État pour s'exonérer de l'accomplissement de ses obligations constitutionnelles, notamment quand cette conduite gouvernementale négative peut conduire à l'annulation voire à l'annihilation de droits constitutionnels imprégnés d'un sens de fondamentalité essentielle.

Il en résulte que l'organisme décisoire maximum du système juridique brésilien, dans sa fonction essentielle de gardien de la Constitution (préservation du code Recht/ Unrecht [Constitutionnel/Inconstitutionnel]) ne comprend pas comme partie intégrante de la réflexivité du droit social à la santé, en cas de danger de mort, la réserve du possible. Sur cette même voie, la Constitution garantit le fonctionnement de l'économie quand elle renie l'appropriation de la réserve du possible par le système juridique.

Il convient de souligner que, à tous les niveaux organisationnels du Pouvoir judiciaire brésilien, comme nous l'avons montré, la réserve du possible et le droit à la santé - lié au droit à la vie - sont considérés comme incompatibles. Il en résulte donc que l'autopoïèse spécifique du Droit passe par l'inapplicabilité de la théorie en question dans les cas relevant du droit social et fondamental à la santé en liaison au danger de mort.

\section{Considérations finales}

L'analyse de la théorie des systèmes sociaux autopoïétiques de Luhmann renforce le fait que ces deux systèmes (droit et économie) doivent maintenir leur propre autopoïèse (et non pas celle d'autres systèmes). Le Droit désire réaffirmer le Droit ou le non Droit comme Droit. Dans cette communication entre systèmes, l'on se rend clairement compte que chacun d'entre eux a une fonction spécifique. 
Cependant, selon les arguments présentés, la réserve du possible et le système économique partent de la même prémisse: la pénurie de ressources. Dans le premier cas, elle oriente la mise à effet des droits sociaux fondamentaux, en les limitant; dans le deuxième, elle se dirige au système économique de manière généralisée. Toutefois, tous deux coïncident dans leur logique. Donc, pour une question de rationalité systémique, la théorie de la réserve du possible fait partie intégrante du système économique et il est donc naturel qu'elle exerce une pression par rapport au système juridique.

Par ailleurs, au vu de ce qui a été exposé, quand l'autopoïèse du système économique devient l'autopoïèse d'un autre système, tous deux perdent leur fonctionnalité et leur raison d'être sociale. L'avertissement de Jossep est éclairant:

Capitalists do not represent the interests of authority of society they are only concerned for their own and other's capacities to make payments - and the political system is excluded from the economy through the closure of the payments system.*

Ainsi, pour ce qui est de sa récursivité interne, il est loisible d'affirmer que l'ouverture cognitive propre du système juridique, lorsqu'elle reçoit la communication issue du système économique (réserve du possible), chasse ce bruit ${ }^{35}$ quand le bien qu'est la vie est en jeu. En effet, au Brésil, l’organisation du Droit, dans son caractère central décisoire, nie, dans ses plus diverses instances - aussi bien au centre que dans la périphérie -, la possibilité d'appliquer la réserve du possible dans cette hypothèse. Comme l'explicite Teubner: ${ }^{36}$ Les «règles du jeu» économiques, malgré tous les empiètements, ne sont pas identiques à des normes juridiques. Pour que le système social continue à maintenir les attentes sur des niveaux socialement acceptables, il est impératif que cette affirmation soit retraitée dans le système juridique.

Voilà pourquoi, nous sommes d'accord avec $\operatorname{Neves}^{37}$ quant au fait que les arguments possibles pour réaliser la redondance nécessaire à la continuité du système juridique passent, au Brésil, par la réaffirmation de l'État démocratique de droit consacré dans la Constitution fédérale de 1988. La cause déjuridifiante de la Constitution (empiètement d'autres codes) pourrait ainsi être mise en équation, en une réalisation du sens du texte constitutionnel, lequel, pour l'instant, dans les pays périphériques, est différent de celui des pays centraux. 


\section{Referências}

AMARAL, Gustavo. Direito, Escassez e Escolha. Em busca de critérios jurídicos para lidar com a escassez de recursos e as decisões trágicas. Rio de Janeiro: Renovar, 2001. ANDRADE, Paes e BONAVIDES, Paulo. História Constitucional do Brasil. Rio de Janeiro: Paz e Terra, 1991, p. 100-130.

CANADA. Canada Health Act. Minister of Justice; 1985. Disponível em: http://laws. justice.gc.ca/PDF/C-6.pdf

CATTANI, Antonio David. Desigualdades Socioeconômicas Brasil/Canadá: um estudo a partir de estudos extremos. Interfaces Brasil Canadá, n. 6. 2006, p. 49-73.

CLAM, Jean. Monetarização, Generalização da Cobiça e Paradoxo do Direito. In: ARNAUD, André-Jean. LOPES JR., Dalmir. Niklas Luhmann: do sistema social à sociologia jurídica. Rio de Janeiro: Lumen Juris, 2004.

CLAM, Jean. Questões Fundamentais de uma Teoria da Sociedade. Contingência, Paradoxo, Só-Efetuação. São Leopoldo: Unisinos, 2006.

CORSI, G.; ESPOSITO, E.; BARALDI, C.Glosario sobre la Teoría Social de Niklas Luhmann. ITESO: México; Anthropos: Barcelona, 1996.

DE GIORGI, Rafaelle. Direito, Democracia e Risco: vínculos com o futuro. Porto Alegre : SAFE, 1998.

DOS SANTOS, Sandro Schmitz. Direito Canadense: algumas particularidades. Interfaces Brasil Canadá, n. 7, 2007, p. 233-250.

HUTTER, Michael. How the Economy Talks The Law into Co-Evolution: An Exercise in Autopoietic Social Theory. In: TEUBNER, Gunther; FEBBRAJO, Alberto. State, Law and Economic as Autopoietic Systems. Regulation and Autonomy in a New Perspective. Milan, Dott. A. Giuffrè Editore, 1992.

JESSOP, Bob. The Economy, The State, and The Law : Theories of Relative Autonomy and Autopoietic Closure. In: TEUBNER, Gunther; FEBBRAJO, Alberto. State, Law and Economic as Autopoietic Systems. Regulation and Autonomy in a New Perspective. Milan, Dott. A. Giuffrè Editore, 1992.

KRELL, Andreas J. Direitos Sociais e Controle Judicial no Brasil e na Alemanha: os (des)caminhos de um direito constitucional “comparado”. Porto Alegre : SAFE, 2002.

LEAL, José Roberto; CABRAL, Ivone Evangelista; PERREAULT, Michel. Experiência Brasil-Canadá no Cuidado Social e na Saúde da Criança com Necessidades Especiais: aproximações e distanciamentos. Interfaces Brasil Canadá, v. 10, n.1, 2010, p. 95-110.

LEIVAS, Paulo Gilberto Cogo. Teoria dos Direitos Fundamentais Sociais. Porto Alegre: Livraria do Advogado, 2006.

LUHMANN, Niklas. Das Recht der Gesellschaft. Frankfurt: Suhrkamp, 1997.

LUHMANN, Niklas. Politics and Economy. Thesis Eleven, Number 53, 1998.

Interfaces Brasil/Canadá. Florianópolis/Pelotas/São Paulo, v. 17, n. 2, 2017, p. 130-148. 
SAGE: London.

LUHMANN, Niklas. Teoria Política en el Estado de Bienestar.2 ${ }^{a}$ reimpresión. Madrid: Alianza Editorial, 1997.

NEVES, Marcelo. Entre Têmis e Leviatã : uma relação difícil. São Paulo: Martins Fontes, 2007.

NEVES, Marcelo. Verfassung und Positivität des Rechts in der peripheren Moderne: eine theoretische Betrachtung und eine Interpretation des Falls Brasilien. Duncker u.H., Bln. Broschiert, 1992.

POSNER, Richard. Economic Analysys of Law. New York: Aspen Law \& Business, 2002.

ROCHA, L; SCHWARTZ, G; CLAM; J. Introdução à Teoria do Sistema Autopoiético do Direito. Porto Alegre: Livraria do Advogado, 2005.

TEUBNER, Gunther. Regimes de Produção Idiossincráticos: Sobre a Co-Evolução da Economia e do Direito nas Varities of Capitalism. In: . Direito, Sistema e Policontexturalidade. Piracicaba: Unimep, 2005.

TEUBNER, Gunther. The Anonymous Matrix: Human Rights Violation by "Private" Transnational Actors. The Modern Law Review Limited. Blackwell: Oxford, 2006.

TRINDADE, André. Os Direitos Fundamentais em uma Perspectiva Autopoiética. Porto Alegre: Livraria do Advogado, 2006.

TANDON, Ajay; MURRAY, Christopher JL; LAUER, Jeremy A; EVANS, David B; Measuring Overall Health System Perfomance for 191 Countries. GPE Discussion Paper Series: No. 30 EIP/GPE/EQC World Health Organization. Disponível em http:// www.who.int/healthinfo/paper30.pdf. Acessado em 23/07/2017, às 10 horas e 31 minutos.

\section{Notes}

${ }^{1}$ Professeur universitaire (Centro Universitário Ritter dos Reis - UniRitter, Centro Universitário das Faculdades Metropolitanas Unidas - FMU, Universidade La Salle). Rio Grande do Sul, Brésil. Bourse de Producitvité en Recherche (Nivel 2)-CNPq. germano.schwartz@globo.com

2 Cet après-midi-là, au Congrès national, quand le député Ulysse Guimarães, qui dirigeait les travaux constitutifs, est monté à la tribune pour promulguer la Constitution fédérale du Brésil, il a déclaré, entre autres, qu'il s'agissait d'une Constitution citoyenne, surnom qu'elle a gardé jusqu'à présent.

3 Au Brésil, la dictature militaire a commencé en 1964 et elle a pris officiellement fin au terme du mandat du Général João Batista Figueiredo, en mars 1985.

${ }^{4}$ Les actes de torture n'ont jamais été légalisés au Brésil, même s'ils ont été pratiqués avec plus d'intensité dans les années 70, notamment à partir de la publication de l’Ato Institucional 05/68 (littéralement « Acte institutionnel », nom donné aux décrets promulgués par les gouvernements militaires).

5 Art. $3 \mathrm{e}, \mathrm{CF} / 88$.

${ }^{6}$ Art. 1 er, $\mathrm{CF} / 88$.

7 L'amendement constitutionnel n ${ }^{\circ} 20$, de 1998, en est le meilleur exemple. Il a reformulé le système de prévoyance brésilien en grande partie en fonction du succès chilien (privatiste). En outre, il a rendu plus rigides les règles concernant en particulier l'âge de la retraite et le temps de contribution. Pourquoi ? Parce 
que le gouvernement n'aurait plus de ressources pour supporter les droits sociaux fondamentaux relatifs à la prévoyance des Brésiliens. De nos jours, en 2017, les discours n'ont pas changé et il est fort probable que, pour les mêmes raisons, ces règles déjà modifiées être le soient plus encore.

${ }^{8}$ La possibilité d'accorder le droit de vote aux analphabètes, aux femmes, aux praças-de-pré (soldats), aux religieux soumis à l'obéissance ecclésiastique et aux mendiants était encore exclue.

${ }^{9}$ ANDRADE, Paes e BONAVIDES, Paulo. História Constitucional do Brasil. Rio de Janeiro: Paz e Terra, 1991, pp. 100-130.

${ }^{10}$ Parti communiste du Brésil.

${ }^{11}$ Parti communiste brésilien.

* Les Atos Institucionais, terme traduit littéralement par «Actes institutionnels », sont les décrets que les gouvernements militaires successifs ont promulgués pour légitimer et légaliser leur intervention dans la vie politique.

${ }_{12}$ KRELL, Andreas J. Direitos Sociais e Controle Judicial no Brasil e na Alemanha: os (des)caminhos de um direito constitucional "comparado". Porto Alegre : SAFE, 2002, p. 51.

${ }^{13}$ CORSI, G.; ESPOSITO, E.; BARALDI, C. Glosario sobre la Teoría Social de Niklas Luhmann. ITESO: México ; Anthropos : Barcelona, 1996, p. 69.

${ }_{14}^{14}$ DE GIORGI, Rafaelle. Direito, Democracia e Risco : vínculos com o futuro. Porto Alegre: SAFE, 1998, p. 176.

${ }^{15}$ LUHMANN, Niklas. Politics and Economy. Thesis Eleven, Number 53, 1998. SAGE: London. p. 2.

16 Voir CLAM, Jean. Monetarização, Generalização da Cobiça e Paradoxo do Direito. In: ARNAUD, AndréJean. LOPES JR., Dalmir. Niklas Luhmann: do sistema social à sociologia jurídica. Rio de Janeiro: Lumen Juris, 2004, p. 220-222.

${ }^{17}$ Cf. JESSOP, Bob. The Economy, The State, and The Law: Theories of Relative Autonomy and Autopoietic Closure. In: TEUBNER, Gunther; FEBBRAJO, Alberto. State, Law and Economic as Autopoietic Systems.

Regulation and Autonomy in a New Perspective. Milan, Dott. A. Giuffrè Editore, 1992, p. 228.

${ }^{18}$ LUHMANN, Politics and Economy, 1998, p. 3: "The financial markets participate in all operations involving payment.: they are like the God of the theologians, present everywhere and implied nowhere." (Les marchés financiers participent à toutes les opérations mettant des paiements en jeu: ils sont comme le Dieu des théologiens, présent partout mais impliqués nulle part)

${ }_{19}$ CORSI, ESPOSITO, BARALDI, Glosario...., 1996, p. 67.

${ }^{20}$ Pour une lecture intéressante d'une corrélation entre droits fondamentaux et besoins humains, voir TRINDADE, André. Os Direitos Fundamentais em uma Perspectiva Autopoiética. Porto Alegre: Livraria do Advogado, 2006.

${ }^{21}$ C'est la ligne de pensée défendue par NEVES, Marcelo. Verfassung und Positivität des Rechts in der peripheren Moderne : eine theoretische Betrachtung und eine Interpretation des Falls Brasilien. Duncker u.H., Bln. Broschiert, 1992.

${ }_{22}^{2}$ LUHMANN, Politics and Economy, 1998, p. 4.

${ }^{23}$ L'établissement du système politique comme autopoḯtique est bien souligné in LUHMANN, Niklas. Teoria Política en el Estado de Bienestar. 2a reimpresión. Madrid : Alianza Editorial, 1997.

${ }^{24}$ Voir POSNER, Richard. Economic Analysys of Law. New York : Aspen Law \& Business, 2002.

${ }^{25}$ AMARAL, Gustavo. Direito, Escassez e Escolha. Em busca de critérios jurídicos para lidar com a escassez de recursos e as decisões trágicas. Rio de Janeiro : Renovar, 2001,pp. 141-142.

${ }^{26}$ HUTTER, Michael. How the Economy Talks The Law into Co-Evolution : An Exercise in Autopoietic Social Theory. In: TEUBNER, Gunther ; FEBBRAJO, Alberto. State, Law and Economic as Autopoietic Systems. Regulation and Autonomy in a New Perspective. Milan, Dott. A. Giuffrè Editore, 1992, p. 265.

${ }_{27}$ CLAM, Jean. Questões Fundamentais de uma Teoria da Sociedade. Contingência, Paradoxo, SóEfetuação. São Leopoldo: Unisinos, 2006, pp. 184-185.

${ }^{28}$ LEIVAS, Paulo Gilberto Cogo. Teoria dos Direitos Fundamentais Sociais. Porto Alegre: Livraria do Advogado, 2006, p. 98.

${ }^{29}$ Cf. LUHMANN, Niklas. Das Recht der Gesellschaft. Frankfurt: Suhrkamp, 1997.

${ }^{30}$ Vide LUHMANN, Niklas. Ausdifferenzierung des Rechts. Beiträge zur Rechtssoziologie und Rechtstheorie. Frankfurt: Suhrkamp, 1999, pp. 39-52.

${ }^{31}$ Pour ce qui est des équivalences fonctionnelles appliquées au droit, voir ROCHA, L; SCHWARTZ, G ; CLAM ; J. Introdução à Teoria do Sistema Autopoiético do Direito. Porto Alegre : Livraria do Advogado, 2005.

32 Jugement interlocutoire proféré dans le compte rendu du P. 10501982412 - Comarca de Passo Fundo - RS. 
33 AgRg no REsp 878.441 - RS (2006/0182329-8).

34 ADPF 45 - Relator Ministro Celso de Mello - DJ 04/05/2004, pp. 1212-1213.

* Les capitalistes ne représentent pas les intérêts d'autorité de la société (ils ne soucient que de leur propre capacité à effectuer des paiements et de celle des autres) et le système politique est exclus de l'économie par la fermeture du système de paiements.

35 Un exemple de la doctrine renforce l'argument de l'impossibilité d'appliquer la théorie de la réserve du possible: «La réponse courante basée sur la principiologie de la Charte de 1988 serait: traiter tout le monde! Et si les ressources sont insuffisantes, il faut en puiser dans les enveloppes d'autres secteurs (transport, soutien économique, service de la dette) qui ne sont pas si intimement liés aux droits les plus essentiels de l'homme: sa vie, son intégrité physique et sa santé. Un relativisme dans ce champ peut conduire à des "pondérations" périlleuses ou antihumanistes du type "Pourquoi dépenser de l'argent pour des malades incurables ou en phase terminale?, etc.." KRELL, Direitos Sociais e Controle Judicial..., 2002, pp. 52-53. Voilà pourquoi, en cas de conflit de principes «Le droit fondamental à la santé prédomine sur la compétence budgétaire du législateur». LEIVAS, Teoria dos Direitos Fundamentais Sociais, 2006, p. 118.

36 TEUBNER, Gunther. Regimes de Produção Idiossincráticos: Sobre a Co-Evolução da Economia e do Direito nas Varities of Capitalism. In: 2005, p. 135. . Direito, Sistema e Policontexturalidade. Piracicaba: Unimep,

37 NEVES, Marcelo. Entre Têmis e Leviatã, 2007, p. 197 et suivantes. 